\title{
A Degree Theory for Lagrangian Boundary Value Problems
}

\author{
Ammar Alsaedy* \\ Alnahrain University \\ Baghdad, Iraq \\ Nikolai Tarkhanov ${ }^{\dagger}$ \\ University of Potsdam \\ Potsdam, Germany
}

Received 08.05.2019, received in revised form 06.09.2019, accepted 06.11.2019

\begin{abstract}
We study those nonlinear partial differential equations which appear as Euler-Lagrange equations of variational problems. On defining weak boundary values of solutions to such equations we initiate the theory of Lagrangian boundary value problems in spaces of appropriate smoothness. We also analyse if the concept of mapping degree of current importance applies to Lagrangian problems.
\end{abstract}

Keywords: nonlinear equations, Lagrangian system, weak boundary values, quasilinear Fredholm operators, mapping degree.

Citation: A.Alsaedy, N.Tarkhanov, A Degree Theory for Lagrangian Boundary Value Problems, J. Sib. Fed. Univ. Math. Phys., 2020, 13(1), 5-25. DOI: 10.17516/1997-1397-2020-13-1-5-25.

\section{Introduction}

Distribution theory steams from weak solutions of linear differential equations and it is hardly efficient for nonlinear equations. The use of distributions is actually difficult in linear boundary value problems, for no canonical duality theory is available for manifolds with boundary $\mathcal{X}$. The scale of Sobolev-Slobodetskij spaces $W^{s, p}(\mathcal{X})$ makes it possible to consider the restrictions of functions to the boundary surface, however, these latter are defined only if $s-1 / p>0$. To go beyond this range, one applies integral equalities obtained by manipulation of the Green formula. The study of general boundary value problems for differential equations in Sobolev-Slobodetskij spaces of negative smoothness goes back at least as far as [22].

For a boundary value problem, the Green formula is determined uniquely up to the counterpart of boundary data within the entire Cauchy data, see [26, 9.2.2]. This allows one to avoid much ambiguity in the choice of formal adjoint boundary value problem and to set up duality. As a result one is in a position to introduce weak solutions of the boundary value problem, see for instance Section 9.3.1 ibid. and elsewhere. The Cauchy data of a weak solution to an overdetermined elliptic system in the interior of $\mathcal{X}$ are proved to possess weak boundary values at $\partial \mathcal{X}$ if and only if the solution is of finite order of growth near the boundary surface, see [26, 9.3.6].

When considering a boundary value problem for a nonlinear equation, one has no good guide to an appropriate concept of weak solution. Perhaps one has to pass to the linearised problem. In any case the definition of a weak solution is implicitly contained in the variational setting of the boundary value problem. If the problem itself fails to be Lagrangian, it can be relaxed to variational one. It is just the task of experienced researcher to recover the concept of weak solution in the variational formulation, see [2].

\footnotetext{
*ammar.alsaedy@ymail.com

†tarkhanov@math.uni-potsdam.de

(c) Siberian Federal University. All rights reserved
} 
As but one tool of this work we introduce the concept of weak boundary values for solutions of nonlinear differential equations. We restrict the discussion to those equations which appear as Euler-Lagrange equations for a variational problem of minimasing the discrepancy $A u-f$ in the problem of finding a function $u$ in $\mathcal{X}$, such that $A u=f(x, u)$ in $\mathcal{X}$ and $B u=u_{0}$ at $\partial \mathcal{X}$. Here, $A$ is an overdetermined elliptic operator of order one and $B$ is a matrix of functions at $\partial \mathcal{X}$. The direct approach of variational calculus of [17] applies well to search for a solution in the Sobolev spaces $W^{1, p}(\mathcal{X})$ with non-extreme $1<p<\infty$. However, the Euler-Lagrange equations include the boundary condition $B^{*}|A u-f|^{p-2}(A u-f)=0$ at $\partial \mathcal{X}$. The function $|A u-f|^{p-2}(A u-f)$ is of class $L^{p^{\prime}}(\mathcal{X})$, where $1 / p+1 / p^{\prime}=1$, and hence $B^{*}|A u-f|^{p-2}(A u-f)$ has no clear meaning at the boundary. We give this expression a weak meaning using the variational setting and an appropriate Green formula.

On specifying the spaces of weak boundary values one is in a position to consider the nonlinear mapping of Banach spaces or, more generally, Banach manifolds corresponding to the Lagrangian problem. The tangent mapping is a morphism of tangent (Banach) bundles and it is given by the linearisation of the nonlinear mapping at the points of $\mathcal{X}$. The nonlinear mapping is called elliptic if its tangent mapping is elliptic at each tangent space, cf. [20]. In this sense the Lagrangian boundary value problems are never elliptic but for $p=2$, for they degenerate at each boundary point where $A u=f(x, u)$. By a Hodge theory for a nonlinear mapping is meant the Hodge theory for the corresponding morphism of tangent (Banach) bundles. This bundle is Hilbert, if $p=2$, in which case the problem arises if the Hodge decompositions depend continuously on the point of the underlying Hilbert manifold. To treat this problem of differential geometry on Hilbert manifold we exploit the results of [27].

Any Lagrangian boundary value problem proves to be a quasilinear Fredholm mapping. To the best of our knowledge, this class of nonlinear mappings was first introduced in [24]. The quasilinear Fredholm mappings admit a reasonable degree theory elaborated in [9]. As but one consequence of our results we show that the degree theory of [9] applies to the Lagrangian boundary value problems.

\section{Lagrangian boundary value problems}

By Lagrangian boundary value problems are meant those arising as the Euler-Lagrange equations for functionals minimising discrepancy in overdetermined problems.

Let $\mathcal{X}$ be a bounded closed domain with $C^{\infty}$ boundary in $\mathbb{R}^{n}$. Consider the boundary value problem

$$
\begin{cases}A u=f(x, u) & \text { in } \mathcal{X} \\ B u=u_{0} & \text { at } \partial \mathcal{X}\end{cases}
$$

where $A$ is a (possibly, overdetermined) elliptic linear partial differential operator of the first order near $\mathcal{X}, f$ a function of its numerical variables $(x, u) \in \mathcal{X} \times \mathbb{R}^{\ell}$ with values in $\mathbb{R}^{m}$, and $B$ an $\left(\ell^{\prime} \times \ell\right)$-matrix of smooth functions on the boundary of $\mathcal{X}$ whose rank is $\ell^{\prime}$ for all $x \in \partial \mathcal{X}$.

The operator $A$ is given by an $(m \times \ell)$-matrix of scalar differential operators in a neighbourhood $U$ of $\mathcal{X}$, and the principal symbol of $A$ has rank $\ell$ for all $(x, \xi) \in U \times\left(\mathbb{R}^{n} \backslash\{0\}\right)$. Our standing requirement on $f$ is that $u \mapsto f(x, u)$ be a continuous mapping of $W^{1, p}\left(\mathcal{X}, \mathbb{R}^{\ell}\right)$ into $L^{p}\left(\mathcal{X}, \mathbb{R}^{m}\right)$.

Remark 1.1. Classical elliptic boundary value problems correspond to the case $m=\ell$ and $\ell^{\prime}=\ell / 2$.

The most conventional Banach space setting of this problem is $W^{1, p}$, where $1<p<\infty$. Hence, we pick $u_{0}$ in $W^{1-1 / p, p}\left(\partial \mathcal{X}, \mathbb{R}^{\ell^{\prime}}\right)$ and look for a $u \in W^{1, p}\left(\mathcal{X}, \mathbb{R}^{\ell}\right)$ satisfying (1.1). 
If the operator

$$
\mathcal{A}=\left(\begin{array}{c}
A \\
B
\end{array}\right): W^{1, p}\left(\mathcal{X}, \mathbb{R}^{\ell}\right) \rightarrow L^{p}\left(\mathcal{X}, \mathbb{R}^{m}\right) \times W^{1-1 / p, p}\left(\partial \mathcal{X}, \mathbb{R}^{\ell^{\prime}}\right)
$$

has a left parametrix $\mathcal{P}=(G, P)$, then on applying $\mathcal{P}$ to (1.1) from the left we obtain

$$
u=G f(\cdot, u)+P u_{0}+(\mathcal{P} \mathcal{A}-I) u
$$

in $\mathcal{X}$ for all $u \in W^{1, p}\left(\mathcal{X}, \mathbb{R}^{\ell}\right)$ satisfying (1.1). (Note that $\mathcal{A}$ possesses a left parametrix if and only if its null space is finite dimensional and its range is complemented, see [18]. In this case $\mathcal{P} \mathcal{A}-I$ can be thought of as projection onto the null space.) The operator $u \mapsto G \circ f(\cdot, u)$ is known as the Hammerstein operator. If $u \mapsto f(\cdot, u)$ maps $W^{1, p}\left(\mathcal{X}, \mathbb{R}^{\ell}\right)$ compactly into $L^{p}\left(\mathcal{X}, \mathbb{R}^{m}\right)$, then the Leray-Schauder theory applies to equation (1.2). However, the solutions of the latter equation need not satisfy (1.1).

Moreover, if $A$ is overdetermined (i.e. $m>\ell$ ) then there is a nonzero differential operator $A^{1}$, such that $A^{1} A=0$. Then, for the equation $A u=f(\cdot, u)$ to be solvable, it is necessary that $A^{1} f(\cdot, u)=0$ in $\mathcal{X}$ for some function $u \in W^{1, p}\left(\mathcal{X}, \mathbb{R}^{\ell}\right)$. Another obstacle to the existence of solutions of problem (1.1) is possible overdeterminacy of boundary conditions. This is the case, e.g., if $\ell^{\prime}=\ell$, i.e. $B u$ represents the whole Cauchy data of $u$ with respect to $A-f(x, \cdot)$ at the boundary surface $\partial \mathcal{X}$. This gives evidence of replacing the exact equation $A u=f(\cdot, u)$ in $\mathcal{X}$ by minimising the discrepancy $A u-f(\cdot, u)$ in the norm of $L^{p}\left(\mathcal{X}, \mathbb{R}^{m}\right)$. For this purpose, we introduce the functional

$$
I(u)=\int_{\mathcal{X}}|A u-f(x, u)|^{p} d x
$$

whose domain is the affine subspace $\mathcal{D}_{I}$ of $W^{1, p}\left(\mathcal{X}, \mathbb{R}^{\ell}\right)$ consisting of all $u$, such that $B u=u_{0}$ at $\partial \mathcal{X}$. Obviously, every solution of (1.1) minimises (1.3). The converse assertion is not true.

Write $m$ for the infimum of $I(u)$ over $u \in \mathcal{D}_{I}$. In order that $u \in \mathcal{D}_{I}$ may satisfy $I(u)=m$ it is necessary that $u$ would fulfill the so-called Euler-Lagrange equations. We now describe these.

Lemma 1.2. Let $C$ be an $\left(\left(\ell-\ell^{\prime}\right) \times \ell\right)$-matrix $C$ of smooth functions on $\partial \mathcal{X}$, such that

$$
\operatorname{rank}\left(\begin{array}{l}
B(x) \\
C(x)
\end{array}\right)=\ell
$$

for all $x \in \partial \mathcal{X}$. Then there are unique matrices $B^{*}$ and $C^{*}$ of continuous functions on $\partial \mathcal{X}$ with the property that

$$
\int_{\partial \mathcal{X}}\left(\left(B u, C^{*} g\right)_{x}-\left(C u, B^{*} g\right)_{x}\right) d s=\int_{\mathcal{X}}\left((A u, g)_{x}-\left(u, A^{*} g\right)_{x}\right) d x
$$

for all $u \in W^{1, p}\left(\mathcal{X}, \mathbb{R}^{\ell}\right)$ and $g \in W^{1, p^{\prime}}\left(\mathcal{X}, \mathbb{R}^{m}\right)$, where $d s$ is the surface measure on the boundary.

As usual, $A^{*}$ stands for the formal adjoint of the differential operator $A$ in a neighbourhood of $\mathcal{X}$.

Proof. For an explicit construction of matrices $B^{*}$ and $C^{*}$ we refer the reader to [2].

Formula (1.4) is usually referred to as the Green formula. On arguing as in Section 3 of [2] one sees that if functional (1.3) has a local extremum at a function $u \in \mathcal{D}_{I}$ then

$$
\int_{\mathcal{X}}\left(\left(A-f_{u}^{\prime}\right) v,|A u-f|^{p-2}(A u-f)\right)_{x} d x=0
$$


for all $v \in W^{1, p}\left(\mathcal{X}, \mathbb{R}^{\ell}\right)$ such that $B v=0$ at $\partial \mathcal{X}$. Here, $f_{u}^{\prime}$ is the Jacobi matrix of $f(x, u)$ with respect to $u=\left(u_{1}, \ldots, u_{\ell}\right)$, i.e., the $(m \times \ell)$-matrix whose entries are $f_{i, u_{j}}^{\prime}$.

If $g=|A u-f|^{p-2}(A u-f)$ is of class $W^{1, p^{\prime}}\left(\mathcal{X}, \mathbb{R}^{m}\right)$, then we can apply formula (1.4) on the left-hand side and move $A-f_{u}^{\prime}$ from $v$ to $|A u-f|^{p-2}(A u-f)$, thus obtaining

$$
\int_{\partial \mathcal{X}}\left(C v, B^{*} g\right)_{x} d s+\int_{\mathcal{X}}\left(v,\left(A-f_{u}^{\prime}\right)^{*} g\right)_{x} d x=0
$$

for all $v \in W^{1, p}\left(\mathcal{X}, \mathbb{R}^{\ell}\right)$ satisfying $B v=0$ at the boundary. We first choose $v$ to be arbitrary with compact support in the interior of $\mathcal{X}$ and so we conclude by the main lemma of variational calculus that $\left(A-f_{u}^{\prime}\right)^{*} g$ vanishes almost everywhere in $\mathcal{X}$. Hence, the boundary integral is equal to zero for all $v \in W^{1, p}\left(\mathcal{X}, \mathbb{R}^{\ell}\right)$, such that $B v=0$ on $\partial \mathcal{X}$. It is a simple matter to see that the boundary integral actually vanishes for all functions $v \in W^{1, p}\left(\mathcal{X}, \mathbb{R}^{\ell}\right)$. Hence it follows immediately that $B^{*} g=0$ on $\partial \mathcal{X}$.

Lemma 1.3. For the variational problem $I(u) \rightarrow \min$ over $u \in \mathcal{D}_{I}$, Euler-Lagrange's equations just amount to

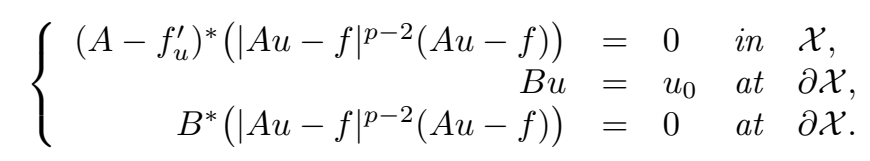

Proof. If $u \in \mathcal{D}_{I}$ and $|A u-f|^{p-2}(A u-f)$ is of class $W^{1, p^{\prime}}\left(\mathcal{X}, \mathbb{R}^{m}\right)$ then this is precisely what has been proved above. For general $u \in \mathcal{D}_{I}$ equalities (1.6) are understood in the weak sense suggested by (1.5). To wit, the differential equation is satisfied in the sense of distributions in the interior of $\mathcal{X}$. The interpretation of the second boundary condition in (1.6) is more sophisticated. This will be discussed in detail in Section 2 .

The differential equation of (1.6) represents a system of $\ell$ second order partial differential equations for $\ell$ unknown functions. The number of boundary conditions just amounts to $\ell$.

Example 1.4. The variational problem of minimising the functional

$$
I(u):=\int_{\mathcal{X}}\left(|d u|^{p}+\left|d^{*} u\right|^{p}\right) d x
$$

over the set of all $i$-forms $u$ of class $W^{1, p}(\mathcal{X})$ with normal part $\nu(u)=u_{0}$ at the boundary leads to the $L^{p}$-setting of the Neumann problem for the de Rham complex in $\mathcal{X}$. To wit,

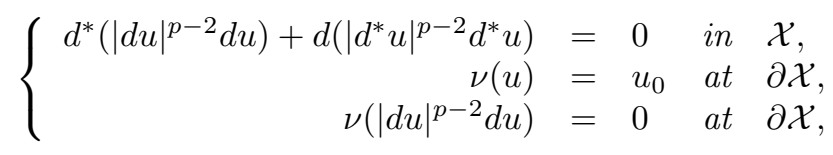

cf. [16].

\section{Weak boundary values}

In (1.6), $u$ is an element of $W^{1, p}\left(\mathcal{X}, \mathbb{R}^{\ell}\right)$, and so $g=|A u-f|^{p-2}(A u-f)$ belongs to $L^{p^{\prime}}\left(\mathcal{X}, \mathbb{R}^{m}\right)$, where $p^{\prime}=p /(p-1)$ is the dual exponent for $p$. Hence, the differential equation $\left(A-f_{u}^{\prime}\right)^{*} g=0$ is readily interpreted in the sense of distributions in the interior of $\mathcal{X}$, just as it comes from (1.5) into consideration. One encounters difficulties in interpreting the equality $B^{*} g=0$ at the boundary surface $\partial \mathcal{X}$, for $g$ is defined almost everywhere in $\mathcal{X}$. To give a meaning to $B^{*} g$ at $\partial \mathcal{X}$, 
we strongly invoke the fact that $g$ satisfies $\left(A-f_{u}^{\prime}\right)^{*} g=0$ weakly in the interior of $\mathcal{X}$. Namely, if $g \in W^{1, p^{\prime}}\left(\mathcal{X}, \mathbb{R}^{m}\right)$, then

$$
\int_{\partial \mathcal{X}}\left(\left(B v, C^{*} g\right)_{x}-\left(C v, B^{*} g\right)_{x}\right) d s=\int_{\mathcal{X}}\left(\left(\left(A-f_{u}^{\prime}\right) v, g\right)_{x}-\left(v,\left(A-f_{u}^{\prime}\right)^{*} g\right)_{x}\right) d x
$$

holds for all $v \in W^{1, p}\left(\mathcal{X}, \mathbb{R}^{\ell}\right)$, which is due to Green formula (1.4). Since $\left(A-f_{u}^{\prime}\right)^{*} g$ vanishes in the interior of $\mathcal{X}$, we may neglect the second term on the right-hand side and use (2.1) to specify both $C^{*} g$ and $B^{*} g$ at the boundary in the general case $g \in L^{p^{\prime}}\left(\mathcal{X}, \mathbb{R}^{m}\right)$.

Definition 2.1. Let $g \in L^{p^{\prime}}\left(\mathcal{X}, \mathbb{R}^{m}\right)$ satisfy $\left(A-f_{u}^{\prime}\right)^{*} g=0$ weakly in the interior of $\mathcal{X}$. Then we define

$$
\int_{\partial \mathcal{X}}\left(\left(v_{0}, C^{*} g\right)_{x}-\left(v_{1}, B^{*} g\right)_{x}\right) d s=\int_{\mathcal{X}}\left(\left(A-f_{u}^{\prime}\right) v, g\right)_{x} d x
$$

for all $v_{0} \in W^{1 / p^{\prime}, p}\left(\partial \mathcal{X}, \mathbb{R}^{\ell^{\prime}}\right)$ and $v_{1} \in W^{1 / p^{\prime}, p}\left(\partial \mathcal{X}, \mathbb{R}^{\ell-\ell^{\prime}}\right)$, where $v \in W^{1, p}\left(\mathcal{X}, \mathbb{R}^{\ell}\right)$ is an arbitrary function satisfying $B v=v_{0}$ and $C v=v_{1}$ at $\partial \mathcal{X}$.

Note that the equalities $B v=v_{0}$ and $C v=v_{1}$ at the boundary surface just amount to

$$
v=\left(\begin{array}{l}
B \\
C
\end{array}\right)^{-1}\left(\begin{array}{l}
v_{0} \\
v_{1}
\end{array}\right)
$$

at $\partial \mathcal{X}$, where the right-hand side belongs to $W^{1 / p^{\prime}, p}\left(\partial \mathcal{X}, \mathbb{R}^{\ell}\right)$. Hence, the existence of a function $v \in W^{1, p}\left(\mathcal{X}, \mathbb{R}^{\ell}\right)$ with the property that $B v=v_{0}$ and $C v=v_{1}$ at $\partial \mathcal{X}$ and

$$
\|v\|_{W^{1, p}\left(\mathcal{X}, \mathbb{R}^{\ell}\right)} \leqslant C\left(\left\|v_{0}\right\|_{W^{1 / p^{\prime}, p}\left(\partial \mathcal{X}, \mathbb{R}^{\prime}\right)}+\left\|v_{1}\right\|_{W^{1 / p^{\prime}, p}\left(\partial \mathcal{X}, \mathbb{R}^{\ell-\ell^{\prime}}\right)}\right)
$$

follows from the Sobolev trace theorem.

Theorem 2.2. Definition 2.1 is correct and specifies the boundary values $C^{*} g$ and $B^{*} g$ in the dual spaces $W^{-1 / p^{\prime}, p^{\prime}}\left(\partial \mathcal{X}, \mathbb{R}^{\ell^{\prime}}\right)$ and $W^{-1 / p^{\prime}, p^{\prime}}\left(\partial \mathcal{X}, \mathbb{R}^{\ell-\ell^{\prime}}\right)$, respectively.

Proof. Suppose $v$ and $w$ are two functions in $W^{1, p}\left(\mathcal{X}, \mathbb{R}^{\ell}\right)$ satisfying $B v=B w$ and $C v=C w$ at $\partial \mathcal{X}$. Set $z=v-w$. Then $z \in W^{1, p}\left(\mathcal{X}, \mathbb{R}^{\ell}\right)$ satisfies $B z=0$ and $C z=0$ at the boundary. By the spectral synthesis theorem for Sobolev spaces, there is a sequence

$$
z_{\nu} \in C_{\text {comp }}^{\infty}\left(\mathcal{X}, \mathbb{R}^{\ell}\right)
$$

which approximates $z$ in the $W^{1, p}\left(\mathcal{X}, \mathbb{R}^{\ell}\right)$-norm. Hence it follows that

$$
\begin{aligned}
\int_{\mathcal{X}}\left(\left(A-f_{u}^{\prime}\right) v, g\right)_{x} d x & =\int_{\mathcal{X}}\left(\left(A-f_{u}^{\prime}\right) w, g\right)_{x} d x+\int_{\mathcal{X}}\left(\left(A-f_{u}^{\prime}\right) z, g\right)_{x} d x= \\
& =\int_{\mathcal{X}}\left(\left(A-f_{u}^{\prime}\right) w, g\right)_{x} d x+\lim _{\nu \rightarrow \infty} \int_{\mathcal{X}}\left(\left(A-f_{u}^{\prime}\right) z_{\nu}, g\right)_{x} d x
\end{aligned}
$$

where the last integral on the right-hand side vanishes, for $g$ satisfies $\left(A-f_{u}^{\prime}\right)^{*} g=0$ weakly in the interior of $\mathcal{X}$. We have thus proved that Definition 2.1 is correct, i.e. it does not depend on the choice of $v$. Finally, combining Definition 2.1 and estimate (2.2) yields

$$
\begin{aligned}
\left|\int_{\partial \mathcal{X}}\left(\left(v_{0}, C^{*} g\right)_{x}-\left(v_{1}, B^{*} g\right)_{x}\right) d s\right| & \leqslant\left\|\left(A-f_{u}^{\prime}\right) v\right\|_{L^{p}\left(\mathcal{X}, \mathbb{R}^{m}\right)}\|g\|_{L^{p^{\prime}}\left(\mathcal{X}, \mathbb{R}^{m}\right)} \\
& \leqslant C\left(\left\|v_{0}\right\|_{W^{1 / p^{\prime}, p}\left(\partial \mathcal{X}, \mathbb{R}^{\ell^{\prime}}\right)}+\left\|v_{1}\right\|_{W^{1 / p^{\prime}, p}\left(\partial \mathcal{X}, \mathbb{R}^{\ell-\ell^{\prime}}\right)}\right)
\end{aligned}
$$

for all $v_{0} \in W^{1 / p^{\prime}, p}\left(\partial \mathcal{X}, \mathbb{R}^{\ell^{\prime}}\right)$ and $v_{1} \in W^{1 / p^{\prime}, p}\left(\partial \mathcal{X}, \mathbb{R}^{\ell-\ell^{\prime}}\right)$, the constant $C$ being independent of $v_{0}$ and $v_{1}$. Hence it follows that $C^{*} g \in W^{-1 / p^{\prime}, p^{\prime}}\left(\partial \mathcal{X}, \mathbb{R}^{\ell^{\prime}}\right)$ and $B^{*} g \in W^{-1 / p^{\prime}, p^{\prime}}\left(\partial \mathcal{X}, \mathbb{R}^{\ell-\ell^{\prime}}\right)$, as desired. 
Thus, for each $u \in W^{1, p}\left(\mathcal{X}, \mathbb{R}^{\ell}\right)$ satisfying $\left(A-f_{u}^{\prime}\right)^{*}\left(|A u-f|^{p-2}(A u-f)\right)=0$ weakly in the interior of $\mathcal{X}$, both $C^{*}\left(|A u-f|^{p-2}(A u-f)\right)$ and $B^{*}\left(|A u-f|^{p-2}(A u-f)\right)$ have weak values at the boundary surface $\partial \mathcal{X}$ which belong to $W^{-1 / p^{\prime}, p^{\prime}}\left(\partial \mathcal{X}, \mathbb{R}^{\ell^{\prime}}\right)$ and $W^{-1 / p^{\prime}, p^{\prime}}\left(\partial \mathcal{X}, \mathbb{R}^{\ell-\ell^{\prime}}\right)$, respectively. This completes, in particular, the result of [23].

For a thorough treatment of weak boundary values of solutions to linear overdetermined elliptic equations we refer the reader to $[26,9.4]$.

\section{Variational boundary value problems after Browder}

By the very nature, the function $\left(A-f_{u}^{\prime}\right)^{*}\left(|A u-f|^{p-2}(A u-f)\right)$ appears as distribution in the interior of $\mathcal{X}$, i.e. as element of

$$
\left(\stackrel{o}{W}^{1, p}\left(\mathcal{X}, \mathbb{R}^{\ell}\right)\right)^{\prime} .
$$

Since $\stackrel{o}{W}^{1, p}\left(\mathcal{X}, \mathbb{R}^{\ell}\right)$ is not dense in $W^{1, p}\left(\mathcal{X}, \mathbb{R}^{\ell}\right)$, the continuous extension of this functional to all of $W^{1, p}\left(\mathcal{X}, \mathbb{R}^{\ell}\right)$ is not uniquely determined. In fact, any continuous extension of $\left(A-f_{u}^{\prime}\right)^{*}\left(|A u-f|^{p-2}(A u-f)\right)$ to a closed subspace $V$ of $W^{1, p}\left(\mathcal{X}, \mathbb{R}^{\ell}\right)$ containing $C^{\infty}$ functions of compact support in the interior of $\mathcal{X}$ with values in $\mathbb{R}^{\ell}$ defines a variational boundary value problem in the sense of [7]. We confine the discussion to (1.5).

Corresponding to the representation (1.5) for the critical points of functional (1.3), we have the nonlinear Dirichlet form $a(u, v)$ defined for all $u$ and $v$ in $W^{1, p}\left(\mathcal{X}, \mathbb{R}^{\ell}\right)$ by

$$
a(u, v)=\left(|A u-f|^{p-2}(A u-f),\left(A-f_{u}^{\prime}\right) v\right),
$$

where $(g, h)$ stands for the natural sesquilinear pairing between $g$ in $L^{p^{\prime}}\left(\mathcal{X}, \mathbb{R}^{m}\right)$ and $h$ in $L^{p}\left(\mathcal{X}, \mathbb{R}^{m}\right)$. By assumption, $a(u, v)$ is well defined for all $u$ and $v$ in $W^{1, p}\left(\mathcal{X}, \mathbb{R}^{\ell}\right)$ and

$$
|a(u, v)| \leqslant c\left(\|u\|_{W^{1, p}\left(\mathcal{X}, \mathbb{R}^{\ell}\right)}\right)\|v\|_{W^{1, p}\left(\mathcal{X}, \mathbb{R}^{\ell}\right)}
$$

by Hölder's inequality, where $c(r)$ is a continuous function of the real variable $r$ depending on $A$ and $f$.

Let $V$ be the closed subspace of $W^{1, p}\left(\mathcal{X}, \mathbb{R}^{\ell}\right)$ that consists of all $v$ satisfying $B v=0$ at the boundary $\partial \mathcal{X}$, and $V^{*}$ be the conjugate space of $V$, i.e. the space of all bounded conjugate linear functionals on $V$. For $w \in V^{*}$ and $v \in V$, the value of $w$ at $v$ is denoted by $(w, v)$. In particular, if $w \in L^{p^{\prime}}\left(\mathcal{X}, \mathbb{R}^{\ell}\right)$, the bounded conjugate linear functional $(w, v)$ on $V$ yields an element of $V^{*}$ which we may again denote by $w$.

We are now in a position to define the variational boundary problem corresponding to $(a, V)$. Denote by $F$ the mapping $V \rightarrow V^{*}$ given by $(F u, v):=a(u, v)$ for all $v \in V$. In particular, we get

$$
F u=\left(A-f_{u}^{\prime}\right)^{*}\left(|A u-f|^{p-2}(A u-f)\right)
$$

in the sense of distributions in the interior of $\mathcal{X}$. Given $w \in V^{*}$, the variational boundary problem corresponding to $(a, V)$ consists in finding $u \in V$ such that $F u=w$. Hence it follows that $F u=w$ holds weakly in the interior of $\mathcal{X}$ and $B u=0$ at the boundary. As usual, in order to include also inhomogeneous conditions $B u=u_{0}$ at $\partial \mathcal{X}$, one solves these first in functions $u \in W^{1, p}\left(\mathcal{X}, \mathbb{R}^{\ell}\right)$ which need not satisfy $F u=w$.

If $u \in V$ satisfies $F u=w$ with $w \in V^{*}$, then $w$ is a relevant extension of the distribution $\left(A-f_{u}^{\prime}\right)^{*}\left(|A u-f|^{p-2}(A u-f)\right)$ in the interior of $\mathcal{X}$ to a continuous linear functional on $V$. Then Definition 2.1 for the weak value of $B^{*} g$ at $\partial \mathcal{X}$ transforms to

$$
\begin{aligned}
-\int_{\partial \mathcal{X}}\left(B^{*} g, v_{1}\right)_{x} d s= & \int_{\mathcal{X}}\left(g,\left(A-f_{u}^{\prime}\right) v\right)_{x} d x-(w, v)= \\
= & a(u, v)-(w, v) \\
& -10-
\end{aligned}
$$


for all $v_{1} \in W^{1 / p^{\prime}, p}\left(\partial \mathcal{X}, \mathbb{R}^{\ell-\ell^{\prime}}\right)$, where $v \in W^{1, p}\left(\mathcal{X}, \mathbb{R}^{\ell}\right)$ is an arbitrary function satisfying $B v=0$ and $C v=v_{1}$ at $\partial \mathcal{X}$. Since $a(u, v)=(w, v)$ for all $v \in V$, it follows that $B^{*} g=0$ at the boundary. Thus, the study of Euler-Lagrange's equations (1.6) can be carried out within the framework of mapping properties of $F: V \rightarrow V^{*}$.

To formulate the hypothesis of our existence theorem, we need an additional concept. Namely, by an admissible lower order operator is meant $u \rightarrow \Delta f(x, u)$, where $\Delta f$ is a continuous function of its numerical arguments satisfying an inequality of the form

$$
|\Delta f(x, u)| \leqslant c\left(\|u\|_{W^{1, p}\left(\mathcal{X}, \mathbb{R}^{\ell}\right)}\right)\left(|u(x)|^{(p-1)+Q}+1\right)
$$

where $0 \leqslant Q<\frac{p^{2}}{n-p}$, if $p \leqslant n$, and $Q=0$, if $p>n$.

Theorem 3.1. Suppose that there exists an admissible lower order operator $\Delta f$ and a continuous function $c(r)$ of the real variable $r$ with $c(r) \rightarrow+\infty$ as $r \rightarrow \infty$, such that

1) If $\Delta a(u, v):=(\Delta f(x, u), v)$ is the nonlinear Dirichlet form corresponding to $\Delta f$, then

$$
\Re(a(u, u-v)-a(v, u-v)+\Delta a(u, u-v)-\Delta a(v, u-v)) \geqslant 0
$$

for all $u$ and $v$ of $V$.

2) For all $u$ in $V$,

$$
\Re a(u, u) \geqslant c\left(\|u\|_{W^{1, p}\left(\mathcal{X}, \mathbb{R}^{\ell}\right)}\right)\|u\|_{W^{1, p}\left(\mathcal{X}, \mathbb{R}^{\ell}\right)} .
$$

Then, for every $w$ in $V^{*}$, the variational boundary problem for $F u=w$ with null $V$-boundary conditions has at least one solution $u$.

Proof. The proof is along the lines of Theorem 1 of [7].

Note that in the case $f \equiv 0$ and $\Delta f=0$ the condition 1 ) is fulfilled. Indeed, we get

$$
\begin{aligned}
\Re & (a(u, u-v)-a(v, u-v))= \\
& =\int_{\mathcal{X}}\left(|A u|^{p}-|A u|^{p-2} \Re(A u, A v)_{x}-|A v|^{p-2} \Re(A v, A u)_{x}+|A v|^{p}\right) d x \geqslant \\
& \geqslant \int_{\mathcal{X}}\left(|A u|^{p}-|A u|^{p-1}|A v|-|A v|^{p-1}|A u|+|A v|^{p}\right) d x \geqslant \\
& \geqslant \int_{\mathcal{X}}\left(|A u|^{p-1}-|A v|^{p-1}\right)(|A u|-|A v|) d x
\end{aligned}
$$

which is obviously nonnegative for all $u, v \in V$. Furthermore, the condition 2) reduces to

$$
\|A u\|_{L^{p}\left(\mathcal{X}, \mathbb{R}^{m}\right)}^{p} \geqslant c\left(\|u\|_{W^{1, p}\left(\mathcal{X}, \mathbb{R}^{\ell}\right)}\right)\|u\|_{W^{1, p}\left(\mathcal{X}, \mathbb{R}^{\ell}\right)}
$$

for all $u \in V$.

\section{Hodge theory for nonlinear mappings}

Let $\mathcal{V}$ and $\mathcal{W}$ be Banach manifolds and $F$ a differentiable mapping of $\mathcal{V}$ to $\mathcal{W}$, i.e. we have a short complex

$$
0 \rightarrow \mathcal{V} \stackrel{F}{\rightarrow} \mathcal{W} \rightarrow 0
$$


Given an arbitrary point $v \in \mathcal{V}$, the tangent mapping $F^{\prime}(v): T_{v} \mathcal{V} \rightarrow T_{w} \mathcal{W}$ is a bounded linear mapping of tangent spaces to $\mathcal{V}$ and $\mathcal{W}$ at $v$ and $w=F(v)$, respectively. These mappings are gathered together to form the Banach bundle morphism

$$
0 \rightarrow T \mathcal{V} \stackrel{F^{\prime}}{\rightarrow} T \mathcal{W} \rightarrow 0
$$

see [27].

Definition 4.1. A differentiable mapping $F: \mathcal{V} \rightarrow \mathcal{W}$ is said to be Fredholm if the linear mappings $F^{\prime}(v): T_{v} \mathcal{V} \rightarrow T_{F(w)} \mathcal{W}$ are Fredholm for all $v \in \mathcal{V}$

By the Hodge theory for the nonlinear mapping $F$ we mean the Hodge theory for the tangent bundle morphism. According to the properties of Fredholm mappings, there are bounded linear projections $P(v)$ and $Q(v)$ in $T_{v} \mathcal{V}$ and $T_{w} \mathcal{W}$, respectively, such that

$$
\begin{aligned}
T_{v} \mathcal{V} & =N\left(F^{\prime}(v)\right) \quad \oplus \quad R(I-P(v)) \\
T_{w} \mathcal{W} & =R(Q(v)) \oplus \quad R\left(F^{\prime}(v)\right),
\end{aligned}
$$

$P(v)$ being a projection onto the finite-dimensional null-space of $F^{\prime}(v)$ and $Q(v)$ being a projection onto a finite-dimensional direct complement of the range of $F^{\prime}(v)$ in $T_{w} \mathcal{W}$.

Using the inverse mapping theorem of Banach we conclude that the restriction of $F^{\prime}(v)$ to $R(I-P(v))$ is an isomorphism of this Banach space onto $R\left(F^{\prime}(v)\right)$. The mapping

$$
\Pi(v)=\left(F^{\prime}(v)\lceil R(I-P(v)))^{-1}(I-Q(v))\right.
$$

is therefore a bounded linear operator from $T_{w} \mathcal{W}$ to $T_{v} \mathcal{V}$ satisfying

$$
\begin{aligned}
& \Pi(v) F^{\prime}(v)=I-P(v), \\
& F^{\prime}(v) \Pi(v)=I-Q(v),
\end{aligned}
$$

i.e. $\Pi(v)$ is a parametrix of $F^{\prime}(v)$ for each $v \in \mathcal{V}$. Note that if $\mathcal{V}$ is contractible then the parametrix $\Pi(v)$ can be chosen to depend continuously on the point $v \in \mathcal{V}$, see [9,27].

If $\mathcal{V}$ and $\mathcal{W}$ are Hilbert manifolds, there is a canonical way for the choice of $P(v)$ and $Q(v)$. Namely, $P(v)$ is the orthogonal projection onto $N\left(F^{\prime}(v)\right)$ and $I-Q(v)$ is the orthogonal projection onto $R\left(F^{\prime}(v)\right)$. By the lemma on the annihilator of the kernel of operator,

$$
\begin{aligned}
R(I-P(v)) & =R\left(F^{\prime}(v)^{*}\right) \\
R(Q(v)) & =N\left(F^{\prime}(v)^{*}\right),
\end{aligned}
$$

where $F^{\prime}(v)^{*}$ is the Hilbert space adjoint for $F^{\prime}(v): T_{v} \mathcal{V} \rightarrow T_{w} \mathcal{W}$. We have thus proved

Theorem 4.2. If $F: \mathcal{V} \rightarrow \mathcal{W}$ is a Fredholm mapping of Hilbert manifolds, then the tangent bundles of $\mathcal{V}$ and $\mathcal{W}$ split as

$$
\begin{aligned}
& T \mathcal{V}=N\left(F^{\prime}\right) \oplus R\left(F^{\prime *}\right), \\
& T \mathcal{W}=N\left(F^{* *}\right) \oplus R\left(F^{\prime}\right) \text {. }
\end{aligned}
$$

These decompositions are scarcely useful to characterise the range of the global nonlinear mapping (4.1).

Example 4.3. Let $F$ be a differentiable selfmapping of $\mathbb{R}^{n}$, such that $\operatorname{det} F^{\prime} \equiv 1$ in all of $\mathbb{R}^{n}$. Then the decompositions of Theorem 4.2 reduce to $T \mathbb{R}^{n}=R\left(F^{*}\right)$ and $T \mathbb{R}^{n}=R\left(F^{\prime}\right)$, however, $F$ need not be surjective in general. This is related to Jacobian problems, cf. [15]. 


\section{Quasilinear Fredholm mappings}

Let $V$ and $W$ be real Banach spaces. Throughout we assume that $V$ is compactly embedded into another Banach space $V^{-}$. When we refer to topological properties of a set $U \subset V$, we will mean the topology induced by $V$, unless we explicitly refer to the topology induced by $V^{-}$.

A mapping $F: V \rightarrow W$ is called quasilinear Fredholm if it can be written in the form

$$
F(v)=L(v) v+C(v)
$$

for $v \in V$, where $L$ is the restriction to $V$ of a continuous mapping $L^{-}$of $V^{-}$into the subset of $\mathcal{L}(V, W)$ consisting of Fredholm operators of index zero, and $C: V \rightarrow W$ is compact. Of course, quasilinear Fredholm mappings need not be differentiable.

Quasilinear Fredholm mappings were introduced in [24] in the study of the nonlinear RiemannHilbert problem. Another typical situation in which quasilinear Fredholm mappings arise quite naturally is the study of the Dirichlet problem for quasilinear elliptic equations. By [3], fully nonlinear elliptic equations with general nonlinear Shapiro-Lopatinskii boundary conditions induce quasilinear Fredholm mappings between appropriate function spaces, provided that the "coefficients" are sufficiently smooth.

If $F: V \rightarrow W$ is any $C^{1}$ mapping, we may write $F$ as $F(v)=L(v) v+F(0)$ for $v \in V$, where $L(v) \in \mathcal{L}(V, W)$ is defined by

$$
L(v)=\int_{0}^{1} F^{\prime}(t v) d t,
$$

which is a curve integral in the space of bounded linear operators from $V$ to $W$. Thus, the algebraic representation of (5.1) is not very restrictive. The crucial point is that each $L(v)$ is a Fredholm operator of index zero and that the family $L(v)$ is defined and depends continuously on $v$ for $v$ belonging to a larger space $V^{-}$in which $V$ is compactly embedded. The latter property implies that $v \mapsto L(v)$ factors through a compact embedding $V \hookrightarrow V^{-}$, and so it is a compact mapping from $V$ to $\mathcal{L}(V, W)$.

We now establish several general properties of quasilinear Fredholm mappings, following [9]. The mapping $L$ is usually referred to as a principal part of $f$. Note that if $L: V \rightarrow \mathcal{L}(V, W)$ is continuous at $v_{0} \in V$ then the mapping of $V$ to $W$ given by $v \mapsto L(v)\left(v-v_{0}\right)$ is Fréchet differentiable at $v_{0}$ and its Fréchet derivative at $v_{0}$ just amounts to $L\left(v_{0}\right)$.

Lemma 5.1. Two principal parts of a quasilinear Fredholm mapping $F: V \rightarrow W$ differ by a family of compact operators.

Proof. Suppose that $F: V \rightarrow W$ is represented by $F(v)=L_{j}(v) v+C_{j}(v)$, for $j=1,2$. Fix $v_{0} \in V$ and set $G_{j}(v)=L_{j}(v)\left(v-v_{0}\right)$ for $v \in V$. As mentioned, we get $G_{j}^{\prime}\left(v_{0}\right)=L_{j}\left(v_{0}\right)$, for $j=1,2$. From the equality of both representations it follows that the difference

$$
G_{1}(v)-G_{2}(v)=-\left(C_{1}(v)-C_{2}(v)\right)-\left(L_{1}(v)-L_{2}(v)\right) v_{0}
$$

is a compact mapping of $V$ to $W$. But the Fréchet derivative of a compact mapping is compact, so that $G_{1}^{\prime}\left(v_{0}\right)-G_{2}^{\prime}\left(v_{0}\right)=L_{1}\left(v_{0}\right)-L_{2}\left(v_{0}\right)$ is compact.

Lemma 5.2. Let $F: V \rightarrow W$ be quasilinear Fredholm and be represented by $F(v)=L(v) v+C(v)$ for $v \in V$. If $F: V \rightarrow W$ is Fréchet differentiable at $v_{0} \in V$, then $F^{\prime}\left(v_{0}\right)-L\left(v_{0}\right)$ is compact.

Proof. Write

$$
R(v)=F(v)-L(v)\left(v-v_{0}\right)
$$

for $v \in V$. The differentiability of $F$ at $v_{0}$ implies that $R^{\prime}\left(v_{0}\right)=F^{\prime}\left(v_{0}\right)-L\left(v_{0}\right)$. Since $R: V \rightarrow W$ is compact, it follows that $F^{\prime}\left(v_{0}\right)-L\left(v_{0}\right)$ is compact, too, as desired. 
So far we have not used the property of $L: V \rightarrow \mathcal{L}(V, W)$ to take on its values in Fredholm operators of index zero. Our next lemma makes use of this property. The Fredholm operators of index zero possess parametrices which are invertible mappings of $W$ onto $V$. We confine ourselves to formulation of this result, referring the reader to [27] and [9] for a proof. Recall that an operator $A \in \mathcal{L}(V, W)$ is Fredholm of index zero if and only if there exists $P \in G L(W, V)$ with $P A-I \in \mathcal{K}(V)$. Let $A(\lambda)$ be a family of Fredholm operators of index zero acting from $V$ to $W$ and continuously depending on a parameter $\lambda \in \Lambda, \Lambda$ being a topological space. By a strong parametrix for $A(\lambda)$ is meant any continuous family $P: \Lambda \rightarrow G L(W, V)$ satisfying $P(\lambda) A(\lambda)-I \in \mathcal{K}(V)$ for all $\lambda \in \Lambda$. In general, a family $A(\lambda)$ has no strong parametrix. For instance, when $\Lambda$ is the unit circle in the plane, the non-existence of strong parametrices for certain continuous families $A(\lambda)$ of Fredholm operators of index zero just amounts to the nontriviality of the Poincaré group of the Fredholm operators of index zero in $\mathcal{L}(V, W)$. However, if $\Lambda$ is a contractible paracompact Hausdorff space, then any continuous family $A(\lambda)$ of $\lambda \in \Lambda$ with values in Fredholm operators of index zero in $\mathcal{L}(V, W)$ possesses a strong parametrix, see Theorem 2.1 of [9] which is referred to as a fundamental result.

Lemma 5.3. Suppose $F: V \rightarrow W$ is a quasilinear Fredholm mapping represented by $F(v)=L(v) v+C(v)$ for $v \in V$. Let $\Pi^{-}: V^{-} \rightarrow G L(W, V)$ be a continuous mapping with the property that $\Pi^{-}(v) L^{-}(v)-I \in \mathcal{K}(V)$ for all $v \in V^{-}$. Then $\Pi^{-}(v) F(v)=v-K(v)$ holds valid for all $v \in V$, where $K: V \rightarrow V$ is a compact mapping.

Proof. We get $\Pi^{-}(v) L^{-}(v)=I-R^{-}(v)$ for $v \in V^{-}$, where $R^{-}: V^{-} \rightarrow \mathcal{K}(V)$ is continuous. Hence,

$$
\begin{aligned}
\Pi^{-}(v) F(v) & =\Pi^{-}(v)(L(v) v+C(v))= \\
& =\left(I-R^{-}(v)\right) v+\Pi^{-}(v) C(v)= \\
& =v-K(v)
\end{aligned}
$$

for all $v \in V$, where $K(v)=R^{-}(v) v-\Pi^{-}(v) C(v)$. Since $V$ is compactly embedded into $V^{-}$and both

$$
\begin{array}{llll}
R^{-}: & V^{-} & \rightarrow & \mathcal{L}(V, W), \\
\Pi^{-}: & V^{-} & \rightarrow & \mathcal{L}(V, W)
\end{array}
$$

are continuous, the compactness of $K: V \rightarrow V$ follows from the compactness of $C: V \rightarrow W$ and of each $R^{-}(v)$ for $v \in V^{-}$.

Theorem 5.4. Let $F: V \rightarrow W$ be a quasilinear Fredholm mapping. Then $F$ can be represented as

$$
F(v)=T^{-}(v)(v-K(v))
$$

for $v \in V$, where $T^{-}: V^{-} \rightarrow G L(V, W)$ is a continuous family of isomorphisms and $K$ is a compact mapping of $V$.

Proof. Write $F$ in the form $F(v)=L(v) v+C(v)$ for $v \in V$. On applying Theorem 2.1 of [9] we choose $\Pi^{-}: V^{-} \rightarrow G L(W, V)$ to be any strong parametrix for the family $L^{-}$. Set

$$
T^{-}(v):=\left(\Pi^{-}(v)\right)^{-1}
$$

for $v \in V^{-}$and use Lemma 5.3 to get (5.2), as desired.

If $A \in \mathcal{L}(V, W)$ is a Fredholm operator of index zero, then the restriction of $A$ to any bounded closed subset of $V$ is proper. The following lemma is a generalisation of this assertion to nonlinear mappings, which is of independent interest as a quite general criterion for establishing properness. 
Lemma 5.5. Assume that $F: V \rightarrow W$ is a quasilinear Fredholm mapping. If $\Sigma \subset V$ is closed and bounded, then $F: \Sigma \rightarrow W$ is proper.

Proof. Let $F: V \rightarrow W$ be represented by (5.1). Then the properness of $F: \Sigma \rightarrow W$ follows from the compactness of the embedding of $V$ into $V^{-}$, the compactness of $C: V \rightarrow W$ and the continuity of $L^{-}: V^{-} \rightarrow \mathcal{L}(V, W)$, together with the properness of $L^{-}(v): \Sigma \rightarrow W$ for each $v \in V^{-}$.

We now turn to the boundary value problem composed in Lemma 1.3. The advantage of using quasilinear Fredholm mappings lies in the fact that they require no linearisation of the problem, which may be cumbersome. To illustrate the results explicitly, we restrict our attention to the case $p=2$, for the theory for $p \neq 2$ does not fit immediately the framework of quasilinear Fredholm operators. If $p=2$ then (1.6) transforms to

$$
\left\{\begin{array}{rlll}
\left(A-f_{u}^{\prime}\right)^{*}(A u-f) & = & 0 & \text { in } \stackrel{\circ}{\mathcal{X}} \\
B u & = & u_{0} & \text { at } \partial \mathcal{X} \\
B^{*}(A-f) & = & 0 & \text { at } \partial \mathcal{X}
\end{array}\right.
$$

cf. [2]. The differential equation of (5.3) is understood in the sense of distributions in the interior of $\mathcal{X}$. While the direct methods of variational calculus apply to look for a solution $u \in H^{1}\left(\mathcal{X}, \mathbb{R}^{\ell}\right)$, direct constructions along more classical lines deal with solutions in $H^{2+s}\left(\mathcal{X}, \mathbb{R}^{\ell}\right)$, where $s=0,1, \ldots$ Under obvious assumption on $f$, the problem corresponds to

$$
\begin{gathered}
H^{s}\left(\mathcal{X}, \mathbb{R}^{\ell}\right) \\
\oplus: H^{s+2}\left(\mathcal{X}, \mathbb{R}^{\ell}\right) \rightarrow \quad H^{s+3 / 2}\left(\partial \mathcal{X}, \mathbb{R}^{\ell^{\prime}}\right) \\
\oplus \\
H^{s+1 / 2}\left(\partial \mathcal{X}, \mathbb{R}^{\ell-\ell^{\prime}}\right)
\end{gathered}
$$

given by $F(u)=L(u) u+C(u)$, where

$$
L(v) u=\left(\begin{array}{c}
A^{*} A u \\
B u \\
B^{*} A u
\end{array}\right), \quad C(u)=\left(\begin{array}{c}
-A^{*} f-\left(f_{u}^{\prime}\right)^{*}(A u-f) \\
0 \\
-B^{*} f
\end{array}\right)
$$

for $v \in H^{1}\left(\mathcal{X}, \mathbb{R}^{\ell}\right)$.

Denote by $H_{B, B^{*} A}^{s+2}\left(\mathcal{X}, \mathbb{R}^{\ell}\right)$ the subspace of $H^{s+2}\left(\mathcal{X}, \mathbb{R}^{\ell}\right)$ that consists of all functions $u \in H^{s+2}\left(\mathcal{X}, \mathbb{R}^{\ell}\right)$ satisfying $B u=0$ and $B^{*}(A u)=0$ at $\partial \mathcal{X}$. Applying Theorem 5 of [1] we conclude that the boundary value problem $L(v)$ is formally selfadjoint relative to the Green formula for the Laplacian $\Delta:=A^{*} A$. Hence it follows that the operator $\left.\Delta: H_{B, B^{*} A}^{s+X^{\prime}}, \mathbb{R}^{\ell}\right) \rightarrow H^{s}\left(\mathcal{X}, \mathbb{R}^{\ell}\right)$ has index zero. We may select a compact operator $K: H_{B, B^{*} A}^{s+2}\left(\mathcal{X}, \mathbb{R}^{\ell}\right) \rightarrow H^{s}\left(\mathcal{X}, \mathbb{R}^{\ell}\right)$ such that $\Delta+K: H_{B, B^{*} A}^{s+2}\left(\mathcal{X}, \mathbb{R}^{\ell}\right) \rightarrow H^{s}\left(\mathcal{X}, \mathbb{R}^{\ell}\right)$ is a bijection. The surjectivity of the boundary operators $\left\{B, B^{*} A\right\}$ then implies that the perturbation of $L(v)$ by $\{K P, 0,0\}$ is bijective, where $P$ is the projection of $H^{s+2}\left(\mathcal{X}, \mathbb{R}^{\ell}\right)$ onto the kernel of $\left\{B, B^{*} A\right\}$. Since the Fredholm index is invariant under compact perturbation, we deduce that $L(v)$ is Fredholm of index zero, cf. Lemma 10.11 of [9]. Hence, $F$ is a quasilinear Fredholm mapping.

\section{Mapping degree of Lagrangian problems}

In [9], an additive integer-valued degree theory for quasilinear Fredholm mappings is constructed. The theory is based upon a modification of the well-known techniques of [14] for 
formulating the solutions of the Dirichlet problem for a quasilinear second order elliptic equation as the zeroes of a compact perturbation of the identity, i.e., fixed points of a compact mapping. Following an idea of [3], it is shown in [9] that general elliptic boundary value problems with sufficiently smooth "coefficients", induce quasilinear Fredholm mappings both in Sobolev and Hölder spaces.

The definition of degree in [9] turns upon first assigning a degree to each linear isomorphism and then extending the degree to general quasilinear Fredholm mappings.

If $V$ and $W$ are finite dimensional of the same dimension, the choice of orientation of $V$ and $W$ defines the determinant $\operatorname{det} T$ for all $T \in G L(V, W)$. Then $\varepsilon: G L(V, W) \rightarrow\{ \pm 1\}$, defined by $\varepsilon(T)=\operatorname{sgn} \operatorname{det} T$, distinguishes the two connected components of $G L(V, W)$. Of course, $\varepsilon(T)$ is the Brower degree of $T$ with respect to the choice of orientations.

If $V=W$ is infinite dimensional, then the group of compact perturbations of the identity in $G L(V, V)$ also has two components, which are distinguished by the function $\varepsilon(T)=(-1)^{N}$ where $N$ is the number of the negative eigenvalues of $T$ counted with their algebraic multiplicities. Obviously, $\varepsilon(T)$ just amounts to the Leray-Schauder degree of $T$.

For general spaces $V$ and $W$ the "group" $G L(V, W)$ may be connected. If we divide $G L(V, W)$ into equivalence classes under the Calkin equivalence relation, to wit $T \sim S$ if $T-S$ is compact, then each equivalence class has two connected components. In fact, if $T-S=K$ then $I-T^{-1} S=T^{-1} K$, and so $T^{-1} S$ is a compact perturbation of the identity. The Leray-Schauder degree of $T^{-1} S$ distinguishes two connected components of the equivalence class indeed. It is reasonable to define the degree so that it would distinguish the components of each Calkin equivalence class. If $T$ and $S$ in $G L(V, W)$ are equivalent, then they lie in the same component of their equivalence class if and only if the Leray-Schauder degree of $T^{-1} S$ is equal to 1 . Accordingly, [9] defines a function $\varepsilon: G L(V, W) \rightarrow\{ \pm 1\}$ to be an orientation provided that $\varepsilon(T) \varepsilon(S)$ just amounts to the Leray-Schauder degree of $T^{-1} S$, if $T, S \in G L(V, W)$ are equivalent. An orientation of $G L(V, V)$ is always required to assign 1 to the identity.

Once an orientation $\varepsilon$ is chosen, the degree of $F$ on an open set $U \subset V$ is defined by

$$
\operatorname{deg}(F, U)=\varepsilon\left(T^{-}(0)\right) \operatorname{deg}(I-K, U, 0),
$$

where $T^{-}$and $K$ are as in (5.2) and $\operatorname{deg}(I-K, U, 0)$ is the Leray-Schauder degree of $I-K$ in $U$ with respect to the value 0 . The right-hand side of (6.1) is independent of representation (5.2).

The degree defined by (6.1) has the usual additivity, existence and Borsuk-Ulam properties, see [9]. If $V=W$ and $G L(V, V)$ is connected, then any integer-valued degree theory on a class of mappings which includes all linear isomorphisms and which coincides with the Leray-Schauder degree on the class of compact perturbation of the identity can neither be homotopy invariant nor can the classical regular value formula hold.

In [10] a rather different construction of mapping degree is given which uses a stronger notion of orientation than the one used in [9]. If $F: V \rightarrow W$ is a $C^{2}$ quasilinear Fredholm map which has 0 as a regular point, then the function $o$ defined by $o(x)=\varepsilon\left(F^{\prime}(x)\right) \sigma\left(F^{\prime} \circ \gamma\right)$, where $\gamma$ is any path between 0 and the regular point $x$ and $\sigma\left(F^{\prime} \circ \gamma\right)$ the parity of the family $F^{\prime}$ along $\gamma$, is an orientation of the map $F$ in the sense of [10]. Moreover, for any admissible set $U$ in $V$, the degree of $F$ with respect to $o$ is

$$
\operatorname{deg}_{o}(F, U, w):=\sum_{x \in F^{-1}(w) \cap U} o(x)
$$

provided that $w \notin F(\partial U)$ is a regular value of $F: U \rightarrow W$. We write it $\operatorname{deg}_{o}(F, U)$ for short, if $w=0$.

A major breakthrough came with the paper [11] which remedied the shortcomings of [10]. Indeed, the theory of [10] has required $C^{2}$ mappings whereas $C^{1}$ mappings would be more natural. The paper [4] is inspired by the approach of [10] though the details are different. The authors 
define the orientation of a linear Fredholm operator $T: V \rightarrow W$ of index zero between Banach spaces as the choice of either of the connected components of the set of all finite rank operators $K$ such that $T+K$ is invertible. They succeed in defining the $\operatorname{degree} \operatorname{deg}(F, U, w)$ whenever $F: U \rightarrow W$ is a $C^{1}$ oriented Fredholm map of index zero between Banach manifolds and $f^{-1}(w)$ is compact, and this degree satisfies the expected properties including invariance under oriented homotopies. For a further progress we refer the reader to $[5,6]$.

We now turn to the Euler-Lagrange equations of Lemma 1.3. In the initial setting the operator

$$
u \mapsto\left(A-f_{u}^{\prime}\right)^{*}\left(|A u-f|^{p-2}(A u-f)\right)
$$

is given the domain $W^{1, p}\left(\mathcal{X}, \mathbb{R}^{\ell}\right)$ and maps it to $\left(W^{o}, p\left(\mathcal{X}, \mathbb{R}^{\ell}\right)\right)^{\prime}$. Our objective is to single out the principal part of the operator containing all second order derivatives of $u$. For this reason our computations will be modulo terms which include the derivatives up to the first order of $u$. Under obvious conditions on $f$ they can be comprehended as nonlinear compact operators in the relevant Banach spaces. We first write

$$
A u=\sum_{j=1}^{n} A^{j} \partial_{j} u+A^{0} u
$$

where $A^{j}$ and $A^{0}$ are $(m \times \ell)$-matrices of smooth functions on $\mathcal{X}$. On using this formula we get

$$
\begin{aligned}
& \left(A-f_{u}^{\prime}\right)^{*}\left(|A u-f|^{p-2}(A u-f)\right)= \\
& =|A u-f|^{p-2} A^{*} A u-\sum_{j=1}^{n} A^{j *}(A u-f) \partial_{j}|A u-f|^{p-2}
\end{aligned}
$$

modulo first order terms. The function $A u$ takes on its values in $\mathbb{R}^{m}$, and we think of $A u$ as an $m$-column with entries $A_{1} u, \ldots, A_{m} u$. By the definition, each $A_{k}$ is an $\ell$-row of scalar partial differential operators of the first order on $\mathcal{X}$. More precisely, we obtain

$$
A_{k} u=\sum_{i=1}^{n} A_{k}^{i} \partial_{i} u+A_{k}^{0} u
$$

for $k=1, \ldots, m$, where $A_{k}^{i}$ and $A_{k}^{0}$ are the $k$ th rows of the matrices $A^{i}$ and $A^{0}$, respectively. Now a trivial verification shows that

$$
\begin{aligned}
\partial_{j}|A u-f|^{p-2} & =\partial_{j}\left(\sum_{k=1}^{m}\left(A_{k} u-f_{k}\right)^{2}\right)^{\frac{p-2}{2}}= \\
& =\frac{p-2}{2}|A u-f|^{p-4}\left(\sum_{k=1}^{m} 2\left(A_{k} u-f_{k}\right) \partial_{j}\left(A_{k} u-f_{k}\right)\right)= \\
& =(p-2)|A u-f|^{p-4}\left(\sum_{k=1}^{m}\left(A_{k} u-f_{k}\right) \sum_{i=1}^{n} A_{k}^{i} \partial_{j} \partial_{i} u\right)
\end{aligned}
$$

modulo nonlinear terms which include the derivatives of $u$ of order not exceeding one. On the other hand, we have

for all $j=1, \ldots, n$, whence

$$
A^{j *}=\left(\begin{array}{lll}
A_{1}^{j *} & \ldots & A_{m}^{j *}
\end{array}\right)
$$

$$
A^{j *}(A u-f)=\sum_{l=1}^{m} A_{l}^{j *}\left(A_{l} u-f_{l}\right) .
$$


Substituting these equalities into (6.2) yields

$$
\begin{aligned}
& \left(A-f_{u}^{\prime}\right)^{*}\left(|A u-f|^{p-2}(A u-f)\right)= \\
& \quad=|A u-f|^{p-2}\left(A^{*} A u-(p-2) \sum_{k, l=1}^{m} \frac{A_{l} u-f_{l}}{|A u-f|} \frac{A_{k} u-f_{k}}{|A u-f|} \sum_{i, j=1}^{n} A_{l}^{j *} A_{k}^{i} \partial_{j} \partial_{i} u\right)
\end{aligned}
$$

modulo nonlinear terms containing the derivatives of $u$ of order $\leqslant 1$. It is easily seen that

$$
-\sum_{i, j=1}^{n} A_{l}^{j *} A_{k}^{i} \partial_{j} \partial_{i} u=A_{l}^{*} A_{k} u
$$

up to terms containing the derivatives of $u$ of order at most one. This gives the final formula

$$
\begin{aligned}
& \left(A-f_{u}^{\prime}\right)^{*}\left(|A u-f|^{p-2}(A u-f)\right)= \\
& \quad=|A u-f|^{p-2}\left(A^{*} A u+(p-2) \sum_{k, l=1}^{m} \frac{A_{l} u-f_{l}}{|A u-f|} \frac{A_{k} u-f_{k}}{|A u-f|} A_{l}^{*} A_{k} u\right)
\end{aligned}
$$

up to terms containing the derivatives of $u$ of order $\leqslant 1$. Formula (6.3) gains in interest if we observe that

$$
A^{*} A=\sum_{k=1}^{n} A_{k}^{*} A_{k}
$$

Remark 6.1. For the classical $p$-Laplace operator in $\mathbb{R}^{n}$ equality (6.3) takes the form

$$
\Delta_{p} u=|\nabla u|^{p-2}\left(-\Delta u-(p-2) \sum_{k, l=1}^{n} \frac{\partial_{l} u}{|\nabla u|} \frac{\partial_{k} u}{|\nabla u|} \partial_{l} \partial_{k} u\right)
$$

modulo terms containing the derivatives of $u$ up to order one.

Summarising we conclude that the operator corresponding to the Euler-Lagrange equations (1.6)

$$
\begin{gathered}
W^{-1, p^{\prime}}\left(\mathcal{X}, \mathbb{R}^{\ell}\right) \\
\oplus \\
W^{1 / p^{\prime}, p}\left(\partial \mathcal{X}, \mathbb{R}^{\ell^{\prime}}\right) \\
\oplus \\
W^{-1 / p^{\prime}, p^{\prime}}\left(\partial \mathcal{X}, \mathbb{R}^{\ell-\ell^{\prime}}\right)
\end{gathered}
$$

can be written in the form $F(u)=L(u) u+C(u)$, where

$$
L(v) u=\left(\begin{array}{c}
|A v-f|^{p-2}\left(A^{*} A u+(p-2) \sum_{\substack{k, l=1 \\
B u}}^{m} \frac{A_{l} v-f_{l}}{|A v-f|} \frac{A_{k} v-f_{k}}{|A v-f|} A_{l}^{*} A_{k} u\right) \\
|A v-f|^{p-2} B^{*} A u
\end{array}\right)
$$

for $v \in W^{1, p}\left(\mathcal{X}, \mathbb{R}^{\ell}\right)$, and $C$ is a nonlinear compact operator. One sees readily that, if $A v-f(\cdot, v)$ vanishes at some point of $\mathcal{X}$, then the boundary value problem $L(v)$ is degenerate.

Theorem 6.2. Let $A v(x)-f(x, v) \neq 0$ for all $x \in \mathcal{X}$. Then the differential equation of $L(v)$ is elliptic in $\mathcal{X}$. 
Proof. The theorem just amounts to saying that the second order partial differential operator

$$
L=A^{*} A+(p-2) \sum_{k, l=1}^{m} \bar{a}_{l} a_{k} A_{l}^{*} A_{k}
$$

is elliptic in $\mathcal{X}$, where $a_{k}=\frac{A_{k} v-f_{k}}{|A v-f|}$ for $k=1, \ldots, m$.

Fix $x \in \mathcal{X}$ and denote by $\sigma(L)=\sigma^{2}(L)(x, \xi)$ the principal symbol of $L$ at a point $(x, \xi) \in T^{*} \mathcal{X}$, where $\xi \in T_{x}^{*} \mathcal{X}$ is different from zero. An easy computation shows that

$$
\sigma(L)=\sum_{k=1}^{m}\left(\sigma\left(A_{k}\right)\right)^{*} \sigma\left(A_{k}\right)+(p-2)\left(\sum_{l=1}^{m} a_{l} \sigma\left(A_{l}\right)\right)^{*}\left(\sum_{k=1}^{m} a_{k} \sigma\left(A_{k}\right)\right),
$$

where $\sigma\left(A_{k}\right)=\sigma^{1}\left(A_{k}\right)(x, \xi)$ is the principal symbol of $A_{k}$ at $(x, \xi)$. The invertibility of $\sigma(L)$ : $\mathbb{R}^{\ell} \rightarrow \mathbb{R}^{\ell}$ will be established once we prove that $(\sigma(L) u, u)>0$ for each nonzero vector $u \in \mathbb{R}^{\ell}$.

We get

$$
(\sigma(L) u, u)=\sum_{k=1}^{m}\left|\sigma\left(A_{k}\right) u\right|^{2}+(p-2)\left|\sum_{k=1}^{m} a_{k} \sigma\left(A_{k}\right) u\right|^{2}
$$

which is obviously nonnegative if $p \geqslant 2$. Furthermore, if $1<p<2$, then using the Cauchy inequality yields

$$
\begin{aligned}
(\sigma(L) u, u) & \geqslant \sum_{k=1}^{m}\left|\sigma\left(A_{k}\right) u\right|^{2}+(p-2) \sum_{k=1}^{m}\left|\sigma\left(A_{k}\right) u\right|^{2} \geqslant \\
& \geqslant 0
\end{aligned}
$$

for $1+(p-2)>0$.

It remains to show that $(\sigma(L) u, u)=0$ for $u \in \mathbb{R}^{\ell}$ implies $u=0$. If $p \geqslant 2$, then from $(\sigma(L) u, u)=0$ it follows that $\sigma\left(A_{k}\right) u=0$ for all $k=1, \ldots, m$. Since the principal symbol mapping of $A$ is injective, we conclude that $u=0$, as desired. The same proof remains valid for $1<p<2$, for if $\sigma(A) u \neq 0$, then $(\sigma(L) u, u)>0$, which is due to (6.4).

Thus, if the system of boundary operators $\left\{B, B^{*} A\right\}$ satisfies the Shapiro-Lopatinskii condition, then $L(v)$ is actually an elliptic boundary value problem. To get rid of degeneracy it suffices to cancel the scalar factor $|A v-f|^{p-2}$, thus obtaining a problem essentially selfadjoint with respect to the Green formula, see Theorem 5 of [1]. Therefore, the theory of [4, 11] still applies to Lagrangian boundary value problems.

\section{Perturbed Dirichlet problem}

In this section we consider the Dirichlet problem for the perturbed Laplace equation and prove criteria which are needed to apply the degree.

Let $\mathcal{X}$ be a bounded closed domain with smooth boundary in $\mathbb{R}^{n}$. Consider the problem

$$
\left\{\begin{array}{rlr}
\Delta u & =f\left(x, u, u^{\prime}\right) & \text { in } \mathcal{X} \\
u & =0 & \text { at } \partial \mathcal{X}
\end{array}\right.
$$

where $f$ is a nonlinear $C^{1}$ function of its numerical arguments $(x, u, p) \in \mathcal{X} \times \mathbb{R} \times \mathbb{R}^{n}$ satisfying

$$
|f| \leqslant C\langle p\rangle^{\gamma}, \quad\left|f_{u}^{\prime}\right| \leqslant C\langle p\rangle^{\gamma}, \quad\left|f_{p}^{\prime}\right| \leqslant C
$$


with $\gamma<1$ and $C$ a constant independent of $x, u$ and $p$. Here, we use the designation $\langle p\rangle=\left(1+|p|^{r}\right)^{1 / r}$ with $r=2$ or with any other $r>0$, for all the expressions are equivalent.

Choose $V:=\stackrel{o}{H^{1}}(\mathcal{X})$ and $W:=H^{-1}(\mathcal{X})$ with norms

$$
\begin{aligned}
\|u\|_{V} & =\left(\int_{\mathcal{X}}\left|u^{\prime}\right|^{2} d x\right)^{1 / 2}, \\
\|f\|_{W} & =\sup _{\|u\|_{V}=1}\left|\int_{\mathcal{X}} f \bar{u} d x\right| .
\end{aligned}
$$

Then, $F(u):=\Delta u-f\left(x, u, u^{\prime}\right)$ maps $V$ continuously into $W$ and it is an elliptic operator.

Lemma 7.1. The Laplace operator $\Delta: V \rightarrow W$ is an isomorphism and $C^{1}$, and so a $C^{1}$ Fredholm operator of index 0.

Proof. To show that $\Delta: V \rightarrow W$ is an isomorphism, note that if $u \in V$ and $\Delta u=0$ then $u=0$, for $u$ is a harmonic function vanishing at the boundary. Thus, $\Delta: V \rightarrow W$ is one to one. We now assume that $f \in H^{-1}(\mathcal{X})$. The equation $\Delta u=f$ for $u \in V$ is understood in the weak sense, i.e., $a(u, v)=f(\bar{v})$ for every $v \in V$, where

$$
a(u, v)=\int_{\mathcal{X}}\left(u^{\prime}, v^{\prime}\right)_{x} d x
$$

stands for the inner product in $V$. By the Riesz representation theorem there is a unique $u \in V$ satisfying $a(u, v)=f(\bar{v})$ for all $v \in V$. Hence it follows that $\Delta: V \rightarrow W$ is onto. Moreover, $\Delta$ is a linear operator and hence $C^{1}$. Thus, $\Delta: V \rightarrow W$ is a $C^{1}$ isomorphism.

Lemma 7.2. Under assumptions (7.2) the Nemytskii map $u \mapsto f\left(x, u, u^{\prime}\right)$ is a $C^{1}$ compact operator.

Proof. We first observe that, for a fixed $u \in V$, the function $x \mapsto f\left(x, u(x), u^{\prime}(x)\right)$ belongs to $L^{p}(\mathcal{X})$ with any $p \geqslant 1$. Consider the map

$$
\stackrel{o}{H^{1}}(\mathcal{X}) \rightarrow L^{2}(\mathcal{X}) \times L^{2}(\mathcal{X})^{n} \stackrel{N_{f}}{\rightarrow} L^{2}(\mathcal{X}) \hookrightarrow H^{-1}(\mathcal{X}),
$$

where by the first arrow is meant the map $u \mapsto\left(u, u^{\prime}\right)$ and by the second arrow the map $\left(u, u^{\prime}\right) \mapsto f\left(x, u, u^{\prime}\right)$. The first map is linear and bounded, hence it is continuous and $C^{1}$. On the other hand, from Theorem 10.58 of [21] and the first inequality of (7.2) it follows that $N_{f}$ is a continuous map from $L^{2}(\mathcal{X}) \times L^{2}(\mathcal{X})^{n}$ to $L^{2}(\mathcal{X})$. And finally the embedding of $L^{2}(\mathcal{X})$ into $H^{-1}(\mathcal{X})$ is also continuous and $C^{1}$. Therefore, (7.3) is a composition of continuous maps and thus is continuous. Moreover, since the last embedding is compact, (7.3) is a compact map from $V$ to $W$. On the other hand, the remaining estimates of (7.2) together with Theorem 10.58 of [21] imply that $N_{f}$ is $C^{1}$, and so (7.3) is $C^{1}$ as composition of $C^{1}$ maps.

We conclude that the map $F: V \rightarrow W$ is of the form $L+C$, where $L u:=\Delta u$ is a linear Fredholm operator of index zero and $C u:=-f\left(x, u, u^{\prime}\right)$ is a compact operator. If $u$ is a smooth function with compact support in the interior of the closed domain $\mathcal{X}$, then

$$
\|\Delta u\|_{W}=\sup _{\|v\|_{V}=1} \int_{\mathcal{X}} \Delta u \bar{v} d x .
$$

On integrating by parts we get

$$
\|\Delta u\|_{W}=\sup _{\|v\|_{V}=1}\left|\int_{\mathcal{X}}\left(u^{\prime}, v^{\prime}\right)_{x} d x\right|=\sup _{\|v\|_{V}=1}\left|(u, v)_{V}\right|
$$


and choosing

$$
v=\frac{u}{\|u\|_{V}}
$$

yields $\|\Delta u\|_{W} \geqslant\|u\|_{V}$. On the other hand, $\|\Delta u\|_{W} \leqslant\|u\|_{V}$, which is clear from the CauchySchwarz inequality. Thus,

$$
\|\Delta u\|_{W}=\|u\|_{V}
$$

which extends by continuity to all functions $u \in \stackrel{o}{H^{1}}(\mathcal{X})$.

If $u \in V$ is a solution of (7.1) then $\Delta u=f\left(x, u, u^{\prime}\right)$, hence

$$
\begin{aligned}
\|u\|_{V} & =\left\|f\left(x, u, u^{\prime}\right)\right\|_{W} \leqslant \\
& \leqslant c\left\|f\left(x, u, u^{\prime}\right)\right\|_{L^{2}(\mathcal{X})}
\end{aligned}
$$

with $c$ a constant independent of $u$. Furthermore, applying the first estimate of (7.2) on $f$ we get

$$
\begin{aligned}
\left\|f\left(x, u, u^{\prime}\right)\right\|_{L^{2}(\mathcal{X})}^{2} & \leqslant C^{2} \int_{\mathcal{X}}\left\langle u^{\prime}\right\rangle^{2 \gamma} d x \leqslant \\
& \leqslant C^{2}\left(\int_{\mathcal{X}} d x\right)^{1-\gamma}\left(\int_{\mathcal{X}}\left\langle u^{\prime}\right\rangle^{2} d x\right)^{\gamma} \leqslant \\
& \leqslant C\left(1+\|u\|_{V}^{2}\right)^{\gamma},
\end{aligned}
$$

where $C$ is a constant independent of $u$ which may be different in diverse applications. Thus,

$$
\|u\|_{V} \leqslant C\left(1+\|u\|_{V}^{2}\right)^{\gamma / 2}
$$

for all $u \in V$ satisfying (7.1). Since the right hand side is a sublinear function of $\|u\|_{V}$, such an a priori estimate occurs only if $\|u\|_{V}$ is bounded, i.e. $\|u\|_{V} \leqslant R$ for some constant $R>0$ independent of $u$.

We may now appeal to the concept of mapping degree to show the existence of a solution to problem (7.1). The specific concept we use here is that of regular point degree clarified in [11, 7.1].

Let $U$ be the ball of radius $2 R$ with centre at the origin in $V$. By Lemmata 7.1 and 7.2, $F$ is a $C^{1}$ map from $\bar{U}$ to $W$. By the above a priori estimate, $F^{-1}(0)$ belongs to the ball $U / 2$, and hence $F$ does not vanish at $\partial U$. It follows that the mapping degree $\operatorname{deg}(F, U)$ is well defined. To compute this degree, we consider the homotopy

$$
F_{t}(u)=\Delta u-t f\left(x, u, u^{\prime}\right)
$$

for $t \in[0,1]$. Obviously, $F_{t}$ is a $C^{1}$ map, for each $t \in[0,1]$, and the same a priori estimate shows that $F_{t}^{-1}(0) \subset U / 2$. Therefore, $F_{t}$ does not vanish at $\partial U$ for all $t \in[0,1]$. Then, the homotopy invariance of the mapping degree implies that $\operatorname{deg}(F, U)=\operatorname{deg}(\Delta, U)$.

By Lemma 7.1, $\Delta: V \rightarrow W$ is a (linear) isomorphism, and so the mapping degree $\operatorname{deg}(\Delta, U)$ is different from zero. This implies immediately that $\operatorname{deg}(F, U) \neq 0$. On using the normalisation property of mapping degree [11] we conclude that the set $F^{-1}(0)$ is nonempty, i.e., problem (7.1) has at least one solution $u \in V$, as desired.

This result extends in an obvious way to the Dirichlet problem for perturbations of the Laplace operator $\Delta=A^{*} A$, where $A$ is a first order overdetermined elliptic differential operator satisfying the uniqueness condition for the local Cauchy problem $(U)_{s}$, see [26]. 


\section{The Dirichlet problem for the $p$-Laplace equation}

In this section we consider the Dirichlet problem for the perturbed $p$-Laplace equation. Let $\mathcal{X}$ be a bounded closed domain with smooth boundary in $\mathbb{R}^{n}$. Consider the problem

$$
\left\{\begin{array}{rlr}
\Delta_{p} u & =f\left(x, u, u^{\prime}\right) & \text { in } \mathcal{X} \\
u & =0 & \text { at } \partial \mathcal{X}
\end{array}\right.
$$

where $\Delta_{p} u:=\nabla^{*}\left(|\nabla u|^{p-2} \nabla u\right)$. The right hand side $f$ is assumed to be a nonlinear $C^{1}$ function of its numerical arguments $(x, u, p) \in \mathcal{X} \times \mathbb{R} \times \mathbb{R}^{n}$ satisfying inequalities (7.2) with some $\gamma<p-1$.

Choose $V:=\stackrel{o}{W}{ }^{1, p}(\mathcal{X})$ and $W:=W^{-1, p^{\prime}}(\mathcal{X})$ with norms

$$
\begin{aligned}
\|u\|_{V} & =\left(\int_{\mathcal{X}}\left|u^{\prime}\right|^{p} d x\right)^{1 / 2}, \\
\|f\|_{W} & =\sup _{\|u\|_{V}=1}\left|\int_{\mathcal{X}} f \bar{u} d x\right|,
\end{aligned}
$$

where $1 / p+1 / p^{\prime}=1$. Then, $F(u):=\Delta_{p} u-f\left(x, u, u^{\prime}\right)$ maps $V$ continuously into $W$ and it is a degenerate elliptic operator.

Lemma 8.1. The map $F: V \rightarrow W$ is $C^{1}$ and it admits a regular point $u_{0}$ in $V$, i.e., $F^{\prime}\left(u_{0}\right) \in$ $\mathrm{GL}(V, W)$.

Proof. Using the chain rule we see that the Fréchet derivative of the $p$-Laplace operator at a point $u_{0} \in V$ is given by

$$
\begin{aligned}
\Delta_{p}^{\prime}\left(u_{0}\right) u & =\nabla^{*}\left(\left|\nabla u_{0}\right|^{p-2}\left(E_{n}+(p-2) \frac{\nabla u_{0}}{\left|\nabla u_{0}\right|}\left(\frac{\nabla u_{0}}{\left|\nabla u_{0}\right|}\right)^{*}\right) \nabla u\right)= \\
& =\nabla^{*}(a(x) \nabla u)
\end{aligned}
$$

for $u \in V$. Note that $a(x)$ is a symmetric $(n \times n)$-matrix with entries in $L^{\frac{p}{p-2}}(\mathcal{X})$. By Theorem $6.2, \Delta_{p}^{\prime}\left(u_{0}\right)$ is a second order elliptic operator away from the critical points of $u_{0}$ in $\mathcal{X}$.

On the other hand, the Fréchet derivative of the map $\hat{f}: V \rightarrow W$ given by $u \mapsto f\left(x, u, u^{\prime}\right)$ is

$$
\hat{f}^{\prime}\left(u_{0}\right) u=f_{u}^{\prime}\left(x, u_{0}, \nabla u_{0}\right) u+f_{p}^{\prime}\left(x, u_{0}, \nabla u_{0}\right) \nabla u
$$

for $u \in V$. The inhomogeneous equation $F^{\prime}\left(u_{0}\right) u=w$ with $w \in W$ just amounts to finding a $u \in V$ which satisfies

$$
\nabla^{*}(a(x) \nabla u)-f_{u}^{\prime}\left(x, u_{0}, \nabla u_{0}\right) u-f_{p}^{\prime}\left(x, u_{0}, \nabla u_{0}\right) \nabla u=w
$$

weakly in $\mathcal{X}$.

We now refer to [12] to see that in any ball around the origin in $V$ there is a function $u_{0}$, such that $F^{\prime}\left(u_{0}\right) u=w$ has a unique solution $u \in V$ for each right hand side $w \in W$. In other words, $F^{\prime}\left(u_{0}\right) \in \mathrm{GL}(V, W)$, i.e., $u_{0}$ is a regular point of $F$, as desired.

If $u$ is a smooth function with compact support in the interior of the closed domain $\mathcal{X}$, then

$$
\left\|\Delta_{p} u\right\|_{W}=\sup _{\|v\|_{V}=1} \int_{\mathcal{X}} \Delta_{p} u \bar{v} d x .
$$

On integrating by parts we get

$$
\left\|\Delta_{p} u\right\|_{W}=\sup _{\|v\|_{V}=1}\left|\int_{\mathcal{X}}\left(\Delta_{p} u, v\right)_{x} d x\right|=\left.\sup _{\|v\|_{V}=1}\left|\int_{\mathcal{X}}\right| \nabla u\right|^{p-2}(\nabla u, \nabla v)_{x} d x \mid
$$


Let

$$
v=\frac{u}{\|u\|_{V}}
$$

then

$$
\begin{aligned}
\int_{\mathcal{X}}|\nabla u|^{p-2}(\nabla u, \nabla v)_{x} d x & =\frac{1}{\|u\|_{V}} \int_{\mathcal{X}}|\nabla u|^{p-2}(\nabla u, \nabla u)_{x} d x= \\
& =\frac{1}{\|u\|_{V}} \int_{\mathcal{X}}|\nabla u|^{p} d x= \\
& =\|u\|_{V}^{p-1}
\end{aligned}
$$

whence $\left\|\Delta_{p} u\right\|_{W} \geqslant\|u\|_{V}^{p-1}$. On the other hand, if $v \in \stackrel{o}{W}^{1, p}(\mathcal{X})$ and $\|v\|_{V}=1$, then

$$
\begin{aligned}
\left.\left|\int_{\mathcal{X}}\right| \nabla u\right|^{p-2}(\nabla u, \nabla v)_{x} d x \mid & \leqslant\|\nabla v\|_{L^{p}(\mathcal{X})}\left(\int_{\mathcal{X}}|\nabla u|^{(p-1) p^{\prime}}(\nabla u, \nabla v)_{x} d x\right)^{1 / p^{\prime}}= \\
& =\|u\|_{V}^{p-1},
\end{aligned}
$$

the first estimate being due to the Hölder inequality. Thus,

$$
\left\|\Delta_{p} u\right\|_{W}=\|u\|_{V}^{p-1}
$$

which extends by continuity to all functions $u \in \stackrel{o}{W}^{1, p}(\mathcal{X})$.

If $u \in V$ is a solution of (8.1) then $\Delta_{p} u=f\left(x, u, u^{\prime}\right)$, hence

$$
\begin{aligned}
\|u\|_{V}^{p-1} & =\left\|f\left(x, u, u^{\prime}\right)\right\|_{W} \leqslant \\
& \leqslant c\left\|f\left(x, u, u^{\prime}\right)\right\|_{L^{p}(\mathcal{X})}
\end{aligned}
$$

with $c$ a constant independent of $u$. Furthermore, on applying the first estimate of (7.2) on $f$ we obtain

$$
\begin{aligned}
\left\|f\left(x, u, u^{\prime}\right)\right\|_{L^{p}(\mathcal{X})}^{p} & \leqslant C^{p} \int_{\mathcal{X}}\left\langle u^{\prime}\right\rangle^{p \gamma} d x \leqslant \\
& \leqslant C\left(\int_{\mathcal{X}}\left\langle u^{\prime}\right\rangle^{p} d x\right)^{\gamma} \leqslant \\
& \leqslant C\left(1+\|u\|_{V}^{p}\right)^{\gamma},
\end{aligned}
$$

where $C$ is a constant independent of $u$ which may be different in diverse applications. Thus,

$$
\|u\|_{V} \leqslant C\left(1+\|u\|_{V}^{p}\right)^{\gamma / p(p-1)}
$$

for all $u \in V$ satisfying (8.1). Since $\gamma<p-1$, the right hand side of this inequality is a sublinear function of $\|u\|_{V}$. On arguing as in Section 7 . we see that there is a constant $R>0$ with the property that $\|u\|_{V} \leqslant R$ is fulfilled for all $u \in V$ satisfying (8.1).

Let $U$ be the ball of radius $2 R$ with centre at the origin in $V$. By Lemma 8.1, $F$ is a $C^{1}$ map from $\bar{U}$ to $W$ and it has a regular point $u_{0} \in U$. By the above a priori estimate, $F^{-1}(0)$ belongs to the ball $U / 2$, and hence $F(u) \neq 0$ for all $u \in \partial U$. It follows that the mapping degree $\operatorname{deg}_{u_{0}}(F, U)$ is well defined, see $[11,7.1]$. To compute this degree, we consider the homotopy

$$
F_{t}(u)=\Delta_{p} u-t f\left(x, u, u^{\prime}\right)
$$

for $t \in[0,1]$. Obviously, $F_{t}$ is a $C^{1}$ map, for each $t \in[0,1]$, and the same a priori estimate shows that $F_{t}^{-1}(0) \subset U / 2$. Therefore, $F_{t}$ does not vanish at $\partial U$ for all $t \in[0,1]$. Then, the homotopy invariance of the mapping degree implies that $\operatorname{deg}(F, U)=\operatorname{deg}\left(\Delta_{p}, U\right)$. 
The mapping $\Delta_{p}: V \rightarrow W$ is well known to be an isomorphism, see for instance [23] and elsewhere. This allows one to conclude that the mapping degree $\operatorname{deg}\left(\Delta_{p}, U\right)$ is different from zero. Hence it follows that $\operatorname{deg}(F, U) \neq 0$, which implies immediately that $F^{-1}(0) \neq \emptyset$. Therefore, problem (8.1) has at least one solution $u \in V$, as desired.

For a deeper discussion of the Dirichlet problem for compact perturbations of the $p$-Laplace equation along more classical lines with $f: \mathcal{X} \times \mathbb{R} \rightarrow \mathbb{R}$ a Carathéodory function we refer the reader to [8].

No attempt has been made here to generalise this result to the Dirichlet problem for the $p$-Laplace operator $u \mapsto A^{*}\left(|A u|^{p-2} A u\right)$ related to a first order overdetermined elliptic differential operator $A$ satisfying the uniqueness condition for the local Cauchy problem $(U)_{s}$.

\section{Conclusion}

As a byproduct of our study of Lagrangian boundary value problems in $\mathcal{X}$ we derived a linearisation of the nonlinear Laplace operator in general outline up to first order terms. It looks like

$$
\Delta(v) u=A^{*} A u+\lambda \sum_{k, l=1}^{m} \frac{A_{l} v-f_{l}(\cdot, v)}{|A v-f(\cdot, v)|} \frac{A_{k} v-f_{k}(\cdot, v)}{|A v-f(\cdot, v)|} A_{l}^{*} A_{k} u,
$$

where $A$ is an $(m \times \ell)$-matrix of first order partial differential operators on $\mathcal{X}$ and $A_{1}, \ldots, A_{m}$ the rows of $A$. If the principal symbol mapping of $A$ is injective away from the zero section of $T^{*} \mathcal{X}$ and $\lambda>-1$, then $\Delta(v)$ is elliptic. This operator is supplied with two boundary operators $B$ and $B^{*} A$ and the problem of solvability of the corresponding boundary value problem in $\mathcal{X}$ is of central interest in the present paper.

Remark 8.2. The operator $\Delta(v)$ is elliptic for all real $\lambda>-1$ and it coincides with $A^{*} A$ for $\lambda=0$. Hence, the index of the boundary value problem $\left\{\Delta(v), B, B^{*} A\right\}$ amounts to that of $\left\{A^{*} A, B, B^{*} A\right\}$ if the boundary operators satisfy the Shapiro-Lopatinskii condition.

The first author gratefully acknowledges the financial support of the Deutscher Akademischer Austauschdienst.

\section{References}

[1] A.Alsaedy, N.Tarkhanov, Journal of Complex Analysis, (2013), Article ID 486934, 11 pages, DOI:10.1155/2013/486934.

[2] A.Alsaedy, N.Tarkhanov, Nonlinear Analysis: Theory, Methods and Applications, 95(2014), 468-482.

[3] A.V.Babin, Math. USSR Sb., 22(1974), 427-454.

[4] P.Benevieri, M.Furi, Ann. Sci. Math. Québec, 22(1998), no. 2, 131-148.

[5] P.Benevieri, M.Furi, M.P.Pera, M.Spadini, Zeitschrift für Analysis und ihre Anwendungen, 22(2003), no. 3, 619-645.

[6] P.Benevieri, M.Furi, Abstract and Applied Analysis, (2006), Article ID 64764, 1-20.

[7] F.E.Browder, Bull. Amer. Math. Soc., 69(1963), no. 4, 862-874.

[8] G.Dinca, P.Jebelean, J.Mawhin, Portugaliae Mathematica, 58(2001), Fasc. 3, 339-378.

[9] P.Fitzpatrick, J.Pejsachowicz, Mem. Amer. Math. Soc., 101(1993), no. 483, 1-131. 
[10] P.Fitzpatrick, J.Pejsachowicz, P.J.Rabier, J. Funct. Anal., 124(1994), 1-39.

[11] P.Fitzpatrick, J.Pejsachowicz, P.J.Rabier, J. d'Analyse Mathématique, 76(1998), 289-319.

[12] A.I.Koshelev, Regularity of Solutions of Elliptic Equations and Systems, Nauka, Moscow, 1986.

[13] M.A.Krasnosel'skii, P.P.Zabreiko, Geometrical Methods in Nonlinear Analysis, Grundlehren Math. Wiss., Vol. 263, Springer-Verlag, Berlin et al., 1984.

[14] J.Leray, J.Schauder, Ann. Sci. Ecóle Norm. Sup., 51(1934), 45-78.

[15] G.H.Meisters, Rocky Mountain J. Math., 12(1982), no. 4, 679-705.

[16] C.B.Morrey, Amer. J. Math., 78(1956), no. 1, 137-170.

[17] C.B.Morrey, Multiple Integrals in the Calculus of Variations, Springer-Verlag, Berlin et al., 1966.

[18] J.I.Nieto, Math. Annalen, 178(1968), 62-77.

[19] L.Nirenberg, Bull. Amer. Math. Soc. (N. S.), 14(1981), 267-302.

[20] R.Palais, Foundations of Global Nonlinear Analysis, Benjamin, New York, 1968.

[21] M.Renardy, R.C.Rogers, An Introduction to Partial Differential Equations, Texts in Applied Mathematics, Springer, 2004.

[22] M.Schechter, Ann. Math., 72(1960), no. 3, 581-593.

[23] I.Shestakov, On the Zaremba problem for the $p$-Laplace operator, In: Complex Analysis and Dynamical Systems V, Contemporary Mathematics, vol. 591, Amer. Math. Soc., Providence, RI, 2013.

[24] A.I.Shnirelman, Math. USSR Sb., 18(1972), 373-396.

[25] E.J.Straube, Ann. Scuola Norm. Super. Pisa, 11(1984), no. 4, 559-591.

[26] N.Tarkhanov, The Cauchy Problem for Solutions of Elliptic Equations, Akademie Verlag, Berlin, 1995.

[27] M.G.Zaidenberg, S.G.Krein, P.A.Kuchment, A.A.Pankov, Russian Math. Surveys, 30(1975), no. $5,115-175$.

\section{Теория степени для лагранжевых краевых задач}

\section{Аммар Аль-Саеди \\ Университет Анахрайна Багдад, Ирак \\ Николай Тарханов \\ Потсдамский университет Потсдам, Германия}

Аннотация. Мы изучаем те нелинейные уравнения с частными производными, которые возникают как уравнения Эйлера-Лагранжа вариационных задач. Определяя слабые граничные значения решений таких уравнений, мы инициируем теорию лагранжевых краевых задач в функциональных пространствах подходящей гладкости. Мы также анализируем, применяется ли современная концепция степени отображения к лагранжевым проблемам.

Ключевые слова: нелинейные уравнения, лагранжева система, слабые граничные значения, квазилинейные операторы Фредхольма, степень отображения. 